\title{
Energy Efficient Congestion Retrieval Strategy for Wireless Sensor Networks
}

\author{
B. Santhana Krishnan \\ Assistant Professor \\ Department of Electrical Engineering \\ Annamalai University
}

\author{
M. Ramaswamy \\ Professor \\ Department of Electrical Engineering \\ Annamalai University
}

\begin{abstract}
Recent advances in Wireless technology ensemble a balanced flow of data load between the sensor nodes and thereby attempt to distribute the energy dissipated throughout the Wireless Sensor Network (WSN). The philosophy orients to create a multipath routing scheme and usher in a new era of undeterred communication between users. It foresees to annihilate the inherent limited energy resource crunch through a prudent operation of the self organized network and ensure an effective data transfer mechanism. The paper strives to develop a cluster based routing strategy with a view to effectively handle the traffic among the chosen paths and thereby endure to reduce congestion. The algorithm evaluated through NS-2 simulation focuses to highlight its ability to accomplish energy efficiency and thereby increase the life time of the network.
\end{abstract}

\section{Keywords}

Wireless Sensor Network, Congestion, CAODV, Load Balancing, Network Life Time

\section{INTRODUCTION}

A sensor network is a group of specialized transducers in a communication infrastructure intended to monitor and record conditions at diverse locations. It consists of multiple detection stations called sensor nodes, each of which is small, lightweight and portable. The transducer in the sensor node generates electrical signals based on sensed physical effects and phenomena. The wireless transceiver receives commands from a central computer and transmits data to that computer. The power for each sensor node is usually derived either from the electric utility or from a battery.

A Wireless Sensor Network (WSN) consists of a large number of nodes that sense the environment, collaboratively process and route the sensor data. [1]. It continues to find a large number of applications that include battlefield and habitat monitoring, tracking of office equipment, and health deployments in the home [2-3]. The sensors extend mobile device functionality, such as providing input to mobile computer applications, receiving commands from mobile computers and consummate sources of information throughout the physical space [4].

The inhospitable conditions may not permit sensors to be deployed uniformly in every corner of the user environment. It results in the sensor networks scaling up in size and requiring a well defined procedure to distribute the network load. The sensors may find it difficult to support long haul communication to reach a command site. It is only by spreading the workload across the sensor network that load balancing averages the energy consumption. The lifespan of the whole sensor network can be extended and in turn serve to reduce congestion and wireless collisions.
A multi-path routing scheme that builds a mesh structure for data reply has been designed [5]. It has been found to reduce the congestion, improve the energy efficiency and the reliability in data delivery. The simulation results have been found to yield a more even distribution of energy consumption among nodes and offer a longer network life time.

An effective reconfiguration algorithm has been proposed to increase the network lifetime by fairly distributing cluster heads $(\mathrm{CHs})$ in wireless sensor networks [6]. The current $\mathrm{CH}$ has been allowed to choose the next $\mathrm{CH}$ based on the number of general nodes (GNs) in a cluster and the number of $\mathrm{CHs}$ within the transmission range. It has been found yield a balanced distribution of GNs in the clusters and facilitate a fair energy consumption of the $\mathrm{CHs}$.

A new load balancing solution suitable for Mobile Adhoc network (MANET) has been proposed [7]. The load balancing has been performed by the source node based on the probe packets sent by the destination node. It has been brought that the connection throughput increases significantly when compared to both proactive Direct Sequence Distance Vector (DSDV) and reactive Adhoc On demand Distance Vector (AODV) routing protocols. A Cluster type structure has been suggested for broadcasting data in MANETs [8]. It has been inferred from simulation that Cluster Networks serve reduce network collision, delay of packet transmission and energy consumption in addition to improving the throughput.

A Secure Load Balancing (SLB) protocol that introduces pseudo-sinks in order to improve data accuracy and lifetime of wireless sensor networks has been presented [9]. It has been found that it increases the data aggregation efficiency of the network, ensures data accuracy and improves the network lifetime due to balanced data rates and enhanced data aggregation ability of the network.

A new e3D Energy-Efficient Distributed Diffusion Routing Algorithm has been introduced to bring out its benefit in terms of system lifetime, power dissipation distribution, cost of synchronization, and simplicity of the methodology [10]. The near optimal performance that has been acclaimed goes to reveal that complex clustering techniques do not end up with perfect balance of load.

A novel altering routing congestion control protocol has been developed for wireless sensor network. It has been focused to build a congestion detection technology with a view to reallocate traffic avoid conflict and enhance data transmission reliability [11]. It has been to found to effectively avoid loss of data during the congestion period and guarantee the reliability of data transmission. 
It is in this direction that a Cluster based Ad hoc On Demand routing (CAODV) pattern suitable for multipath data transfer in a WSN is proposed to accord a congestion management procedure and guarantee an energy efficient mode of operation.

\section{PROBLEM DESCRIPTION}

The primary objective is to balance the load in a WSN through a multipath routing procedure that augurs to reduce the energy consumption and increase the network lifetime. It involves the use of a CAODV technique [12] in its perspective to migrate the data packets between the defined source and destination. The performance is evaluated using NS-2 based simulation over increasing data sizes. The metrics obtained through this approach are to be compared with similar other schemes to illustrate its viability for practical implementation.

\section{SYSTEM MODEL}

A large number of sensor nodes are deployed either inside or very close to it in a WSN displayed through the system model in Figure 1. Each node consists of sensing, data processing, and communication components, to process information before being sent to the remote base station. These sensor nodes can self- organize themselves to form a network and communicate with each other in a wireless manner. Each node possesses control of transmitted power and an Omnidirectional antenna and therefore can adjust the area of coverage through its wireless transmission. The nodes collect audio, seismic and other types of data and collaborate to perform a high level task in a sensor web. The sensor nodes are characterized by a small memory and it is to operate with limited battery power owing to the fact that wireless communications consume significant amounts of battery power. Hence the sensor nodes are required to be energy efficient in transmitting data.

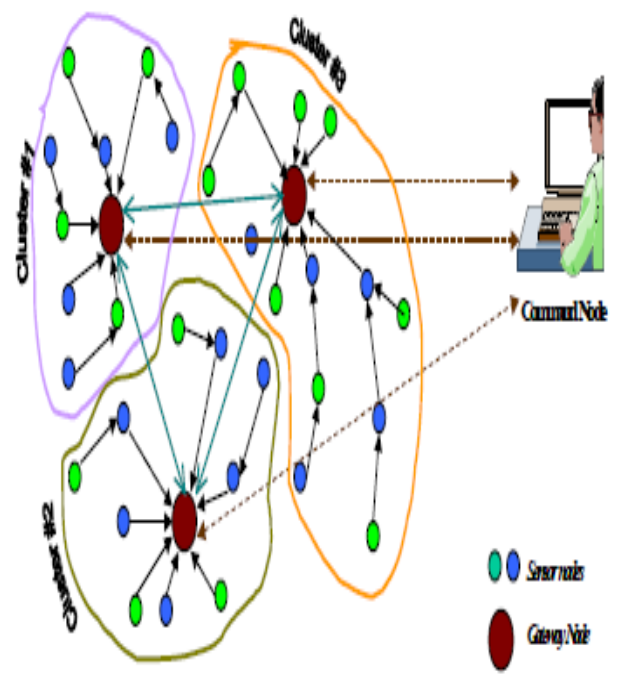

Figure 1. System Model

\section{PROPOSED APPROACH}

The proposed scheme is articulated so as to avail the use of CAODV routing protocol in order to build a load balancing algorithm in a varied traffic platform. It is an on demand algorithm in the sense it creates routes only on specific requests and maintains them as long as they are in use. It is loop-free, self-starting, and uses sequences numbers to ensure the resurgence of routes.
The traffic characteristics of sensor networks vary and may be very different from those of conventional wireless networks. They mainly depend on operational modes that indicate the characteristics and patterns of the measurement and information to be transmitted. The sensor nodes exercise power control in order that they transmit their data directly to the Base Station (BS) or to any other nodes in the network. Further they are assumed to be homogeneous and energy constrained with uniform energy.

An important operation in a sensor network is systematic transfer of data from the field, where each node has a packet of information in each round of communication. The amount of energy spent in transmitting a packet has a fixed cost in electronics and a variable cost that depends on the distance of transmission. Therefore, to conserve energy short distance transmissions are preferred In order to balance the energy spent in the sensor nodes, the nodes should take turns in transmitting to the BS, as this is an expensive transmission. In each round, the data from the different nodes are required to be collected and transmitted to the BS, where the end-user can access the data. A simple approach to accomplish this task is to allow each node to transmit its data directly to the BS. Since the BS is located far away, the cost to transmit to the BS from any node is high, and therefore, the total energy cost per round may turn out to be high.

A group of nodes, each assigned to bear a unique ID number constitutes a cluster. The node with the highest energy level node in the cluster, called the Cluster Head (CH) is characterized with larger resources and generally preferred to collect and merge local traffic and forward it to the base station. The $\mathrm{CH}$ assumes the responsibility to integrate and transmit the cluster node data to the BS during the network operation. The nodes imbibe their ability to communicate with their $\mathrm{CH}$ directly even in the absence of a data exchange among the sensor nodes. The routing scheme incorporates a two stage procedure to involve gather data systematically from the field followed by a reconstruction stage in the event of the energy level of $\mathrm{CH}$ decreases. It follows a ring search mechanism to trace new routes to an unknown destination and proceeds to penetrate large neighborhoods to reach the destination. The algorithm maintains in a table, a track of the node from which the initial request originates. It serves to construct the reverse path through symmetric links to send the reply packet.

The methodology evolves with a choice of a common $\mathrm{CH}$ to traverse a stream of data between the preferred sources to the destinations using as many paths for which it is designed. It is through this node that the transfer is embodied and the neighbors broadcast this packet to its neighbors. The process continues until an intermediate node that carries the recent route information is reached or till it arrives at the destination. The most significant truth is that there is a lifetime associated with each route and if it is not used within its lifetime, it is likely to be eliminated. If the energy level of the $\mathrm{CH}$ declines, it can reinitiate route discovery to the destination. On the other hand if one of the intermediate nodes moves then the neighbor acknowledges the link failure and forges a link failure notification to its upstream neighbors and thereby informs the source upon which the source can reincarnate route discovery.

\section{SIMULATION RESULTS}

The strategy is evaluated when a stream of data are allowed to flow from three different sources to three different destinations through a common $\mathrm{CH}$. The performance is 
measured using a comparison of six performance indices namely Throughput, Energy consumed, Delay, Packet Delivery Ratio (PDR), Packet loss and Number of packets received for three routing protocols.

The network seen in Figure 2 is constructed of randomly distributed fifty sensor nodes in a space of $1000 \mathrm{~m} \mathrm{X} \mathrm{1000m}$ and uses NS-2 simulation for its investigation. The nodes are free from energy dissipation when they are idle or in carrier sensing mode. The algorithm permits the sensed data to move from node to node so that the average energy spent by each node per round is minimized.

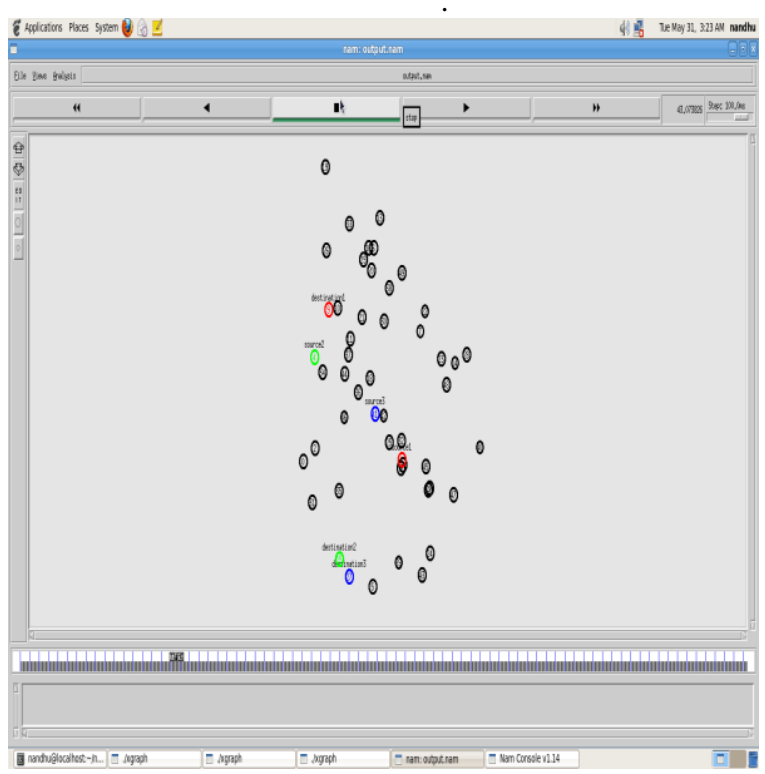

Figure 2. Network Model

The performance indices are computed over a range of two hundred seconds for the chosen source and the defined destination and graphically displayed. The NS -2 graphs obtained for a data size of $1000 \mathrm{Kbps}$ between three source and destination pairs are displayed through Figures 3 to 8. The CAODV extracts the highest throughput than the other two routing patterns (AODV and Improved-AODV) as observed from Figure 3.

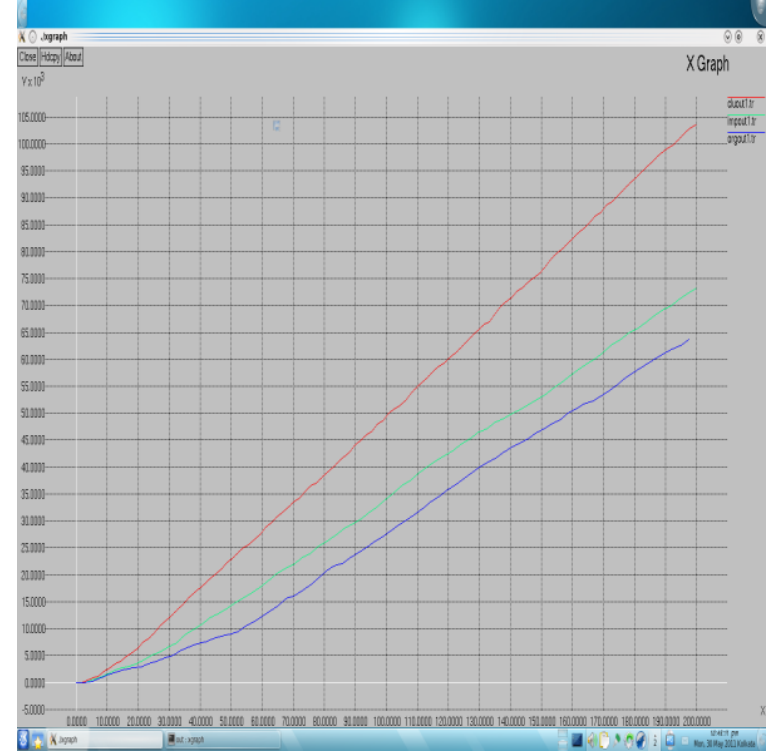

Figure 3. Throughput vs Time

It is observed from Figure 4 that the nodes consume the least energy when data transmission is exercised through CAODV, thereby increasing the lifetime of the network besides minimising the routing overhead.

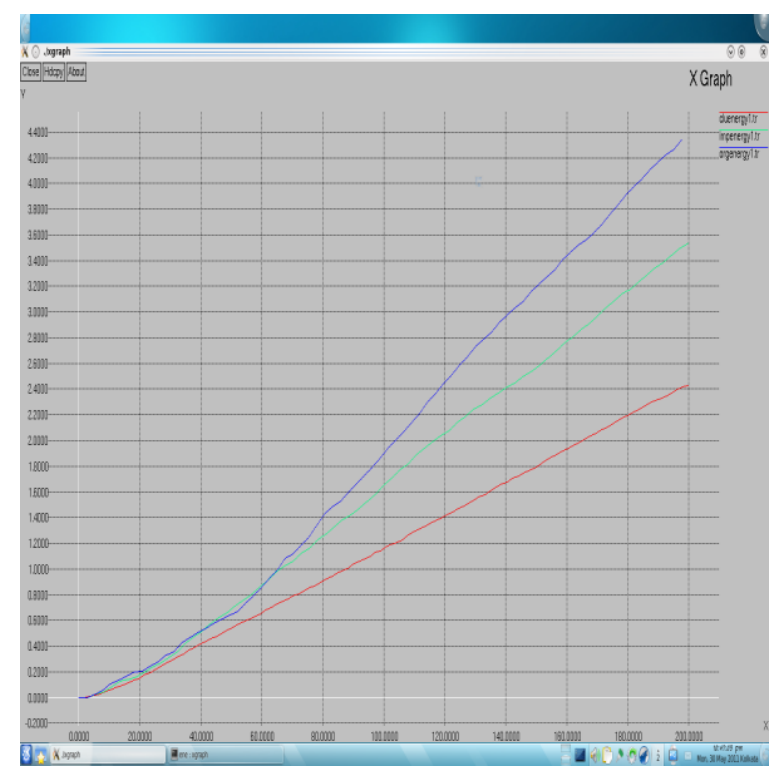

Figure 4. Energy Consumed vs Time

It is significant to observe that there is minimum loss of packets through the use of CAODV as portrayed in Figure 5 and hence it contributes to an increase in the network efficiency. 


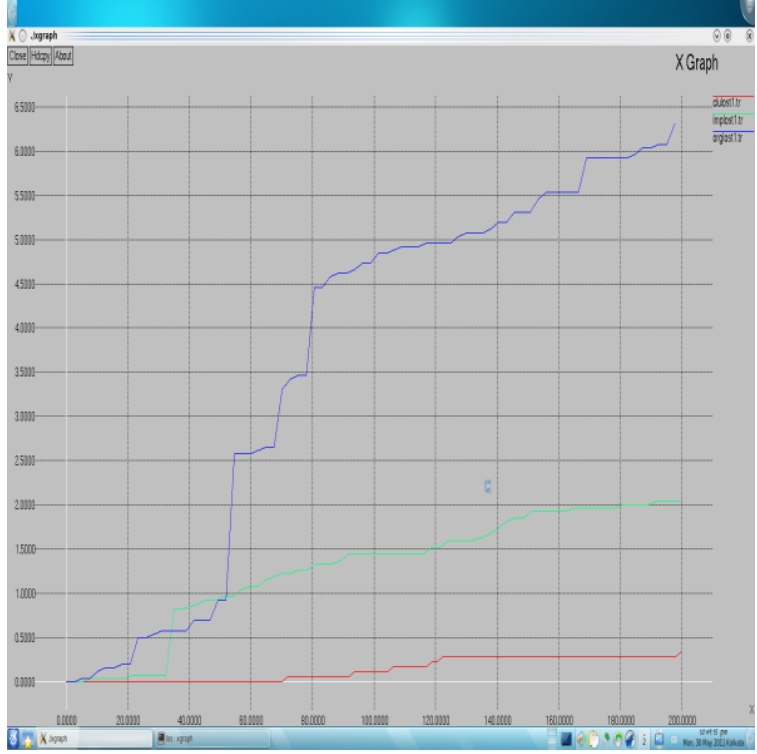

Figure 5. Packet Loss vs Time

The Figure 6 displays the number of packets received with respect to time and it is clearly evident that CAODV tailors the network to transfer the maximum number of packets between the source and the destination.

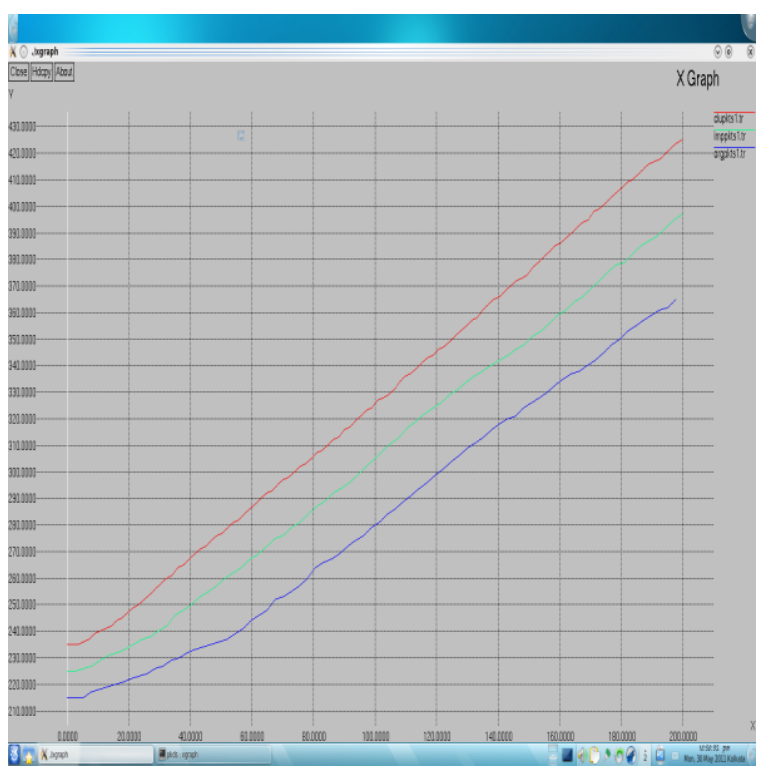

Figure 6. Packets received vs Time

The routing delay of the nodes in the network when it encompasses data transmission is displayed in Figure 7. The Cluster based AODV approach facilitates the data transmission between the source and destination nodes with minimum delay as compared with its counterparts, giving rise to an overall improvement in the performance of the network.

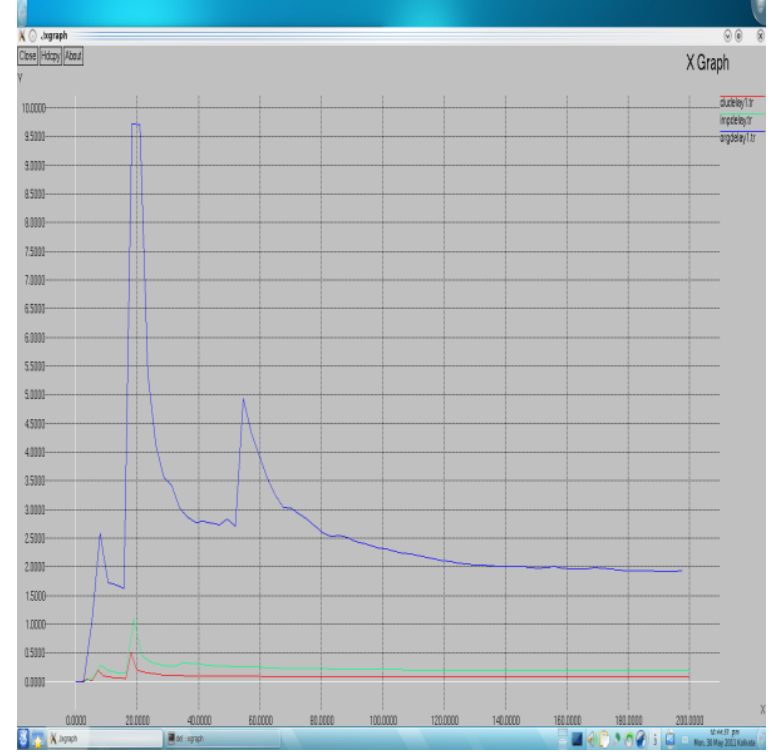

Figure 7. Routing Delay vs Time

The results seen through Figures 8 and 14 are computed for the same performance metrics and displayed through bar charts, when increased packet sizes of data are transmitted between the same three pairs of source and destination. The analysis serves to portray that the algorithm is consistent in its mission and reveals that the protocol is viable for large scale transmission. It follows from Figure 8 that CAODV exhibits a linear increase in throughput over large sizes of data transmission.

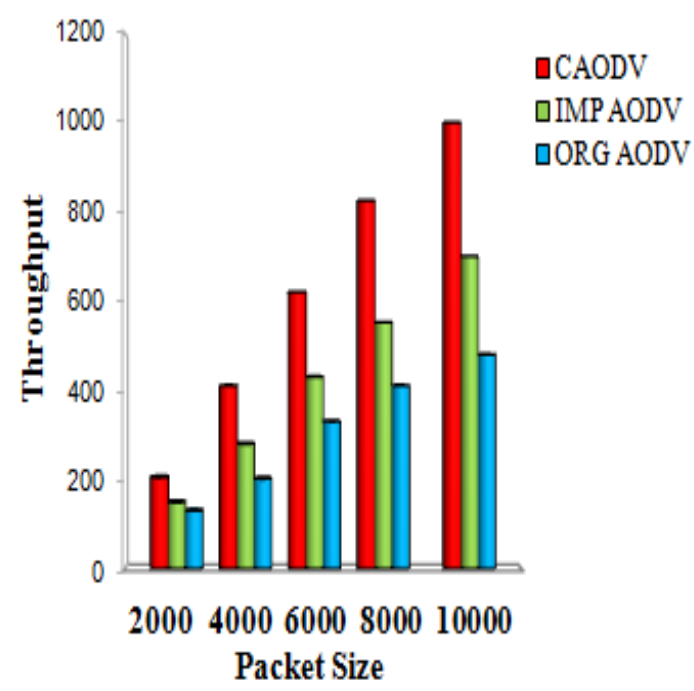

Figure 8. Throughput vs Packet Sizes

The energy consumption of the network apparently increases because of an increase in the size of the packets transfered using CAODV between the chosen source and the destination as seen from Figure 9. It is interesting to observe that the network requires the least energy to transmit the data of same size through the use of the proposed cluster based AODV routing scheme. 


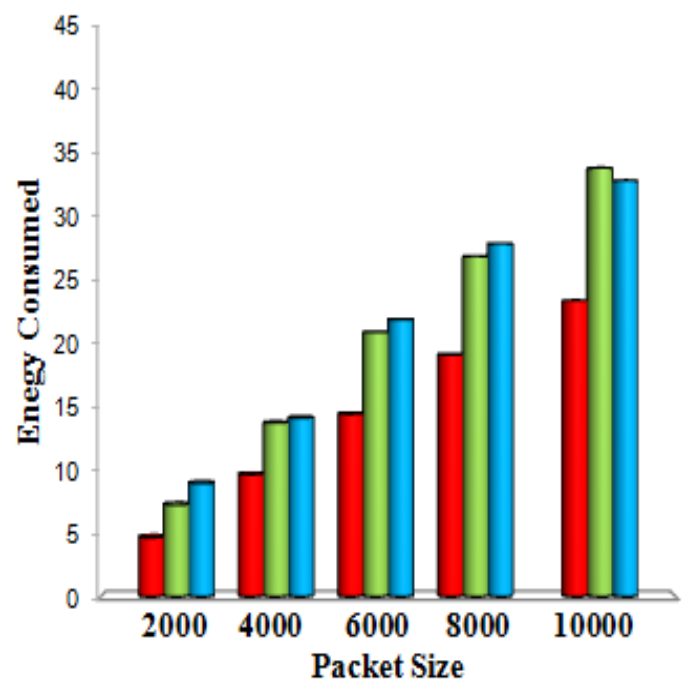

Figure 9. Energy Consumed vs Packet Sizes

It follows from Figure 10 that the network PDR experiences a marginal decline for an increase in the number of transmitted packets. However CAODV stil continues to offer the highest value, which serves to elaborate its energy efficiency and foster the cause of extending the lifetime of the network.

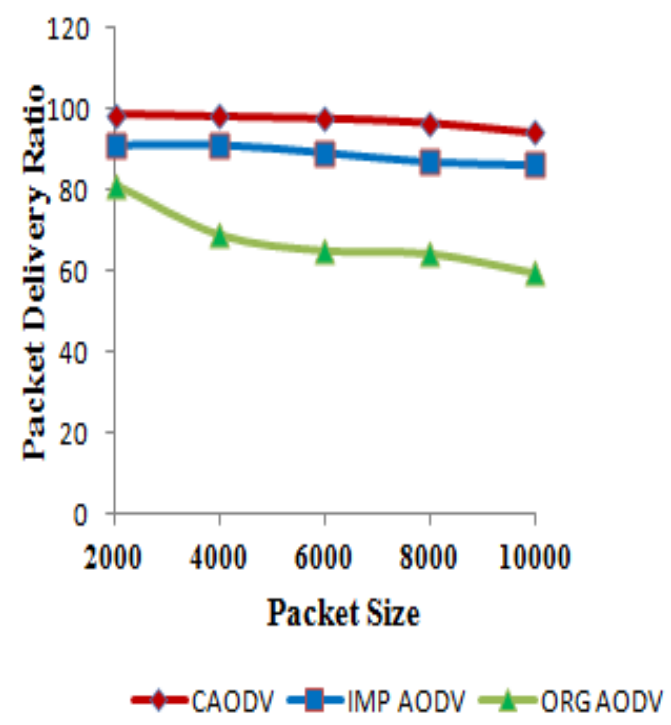

Figure 10. Packet Delivery Ratio vs Packet Sizes

The Figure 11 displays that larger the size of the packets transmitted, higher is the number of packets received. It is evident that this feature of CAODV protocol paves the way to accomplish an overall improvement in the performance of the network.

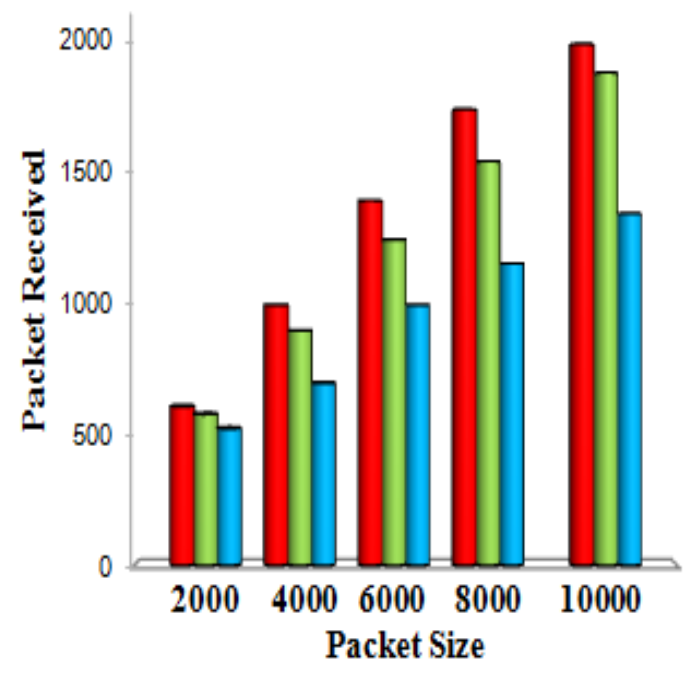

Figure 11. Packets Received vs Packet Sizes

It is noticed that CAODV based data transfer scheme facilitates a gradual decrease in the routing delay and a corresponding fall in routing overhead for an increased packet size trasmission between the source and destinaton nodes under study as depicted in Figure 12, thus enabling a still higher performance of the network. Besides this routing pattern incurs the lowest packet loss as noticed from Figure 13.

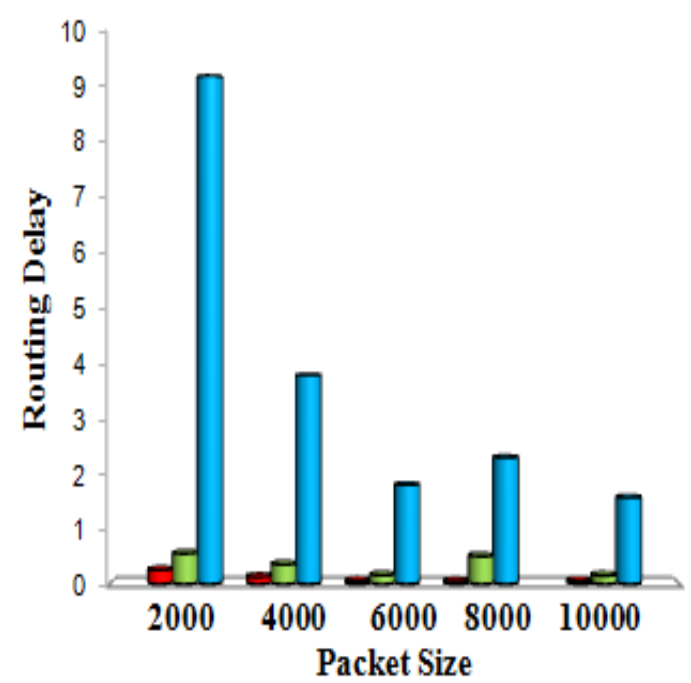

Figure 12. Routing Delay vs. Packet Sizes 


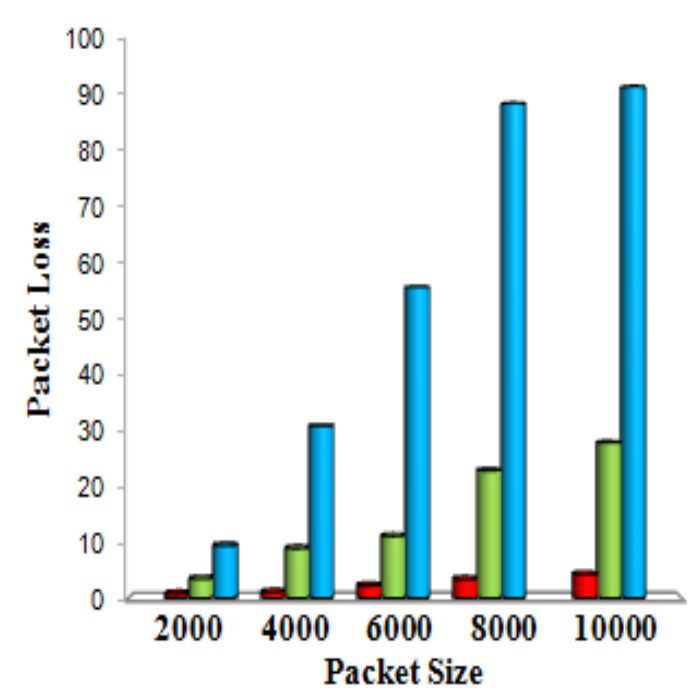

Figure 13. Packet Loss vs. Packet Sizes

The performance of such networks is measured from another important metric Energy*Delay which is shown for varying packet sizes in Figure 14. It can be seen that it is significantly lower for the suggested protocol and thereby helps to realise an economic means of transmission at a reasonably fast rate.

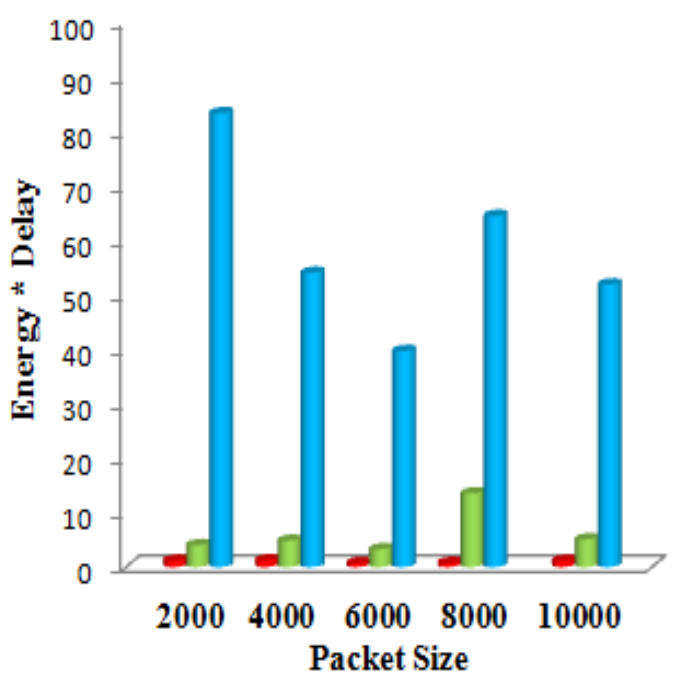

Figure 14. Energy * Delay vs. Packet Sizes

\section{CONCLUSION}

A multi path routing mechanism has been formulated to retrieve congestion in a WSN. It has been articulated through the use of CAODV routing to elicit improved performance indices. The scheme has been designed to exploit an energy efficient methodology and wriggle out the realms of present day requirements. The simulated NS-2 graphs have been espoused to bring out the need for balancing the load in the traffic congested scenario. It has been tailored to extricate the best metrics in terms of higher throughput and number of packets received. The approach has been found to facilitate the procedure with minimum delay and lower associated losses. The CAODV based technique has been found to enjoy resource usage efficiency across the network, exhibiting its suitability for use in practical networks.

\section{ACKNOWLEDGMENT}

The authors thank the authorities of Annamalai University for providing the necessary facilities in order to accomplish this piece of work.

\section{REFERENCES}

[1] I.Akyildiz, W. Su, Y. Sankarasubramaniam, and E.Cayirci. "Wireless Sensor Networks: A Survey", Computer Networks, 38(4), March 2002, pp. 393-422.

[2] Cerpa, J. Elson, D. Estrin, L. Girod, M. Hamilton, and J. Zhao, "Habitat Monitoring: Application Driver for Wireless Communications Technology", Proceedings of the First ACM SIGCOMM Workshop on Data Communications in Latin America, 2001.

[3] D. Estrin, L. Girod, G. Pottie, M. Srivastava , "Instrumenting the world with wireless sensor networks" In Proceedings of the International Conference on Acoustics, Speech and Signal Processing (ICASSP 2001) , Salt Lake City, Utah, May 2001.

[4] Raicu I., Richter O., Schwiebert L., and Zeadally S., "Using Wireless Sensor Networks to Narrow the Gap between Low-Level Information and ContextAwareness," ISCA Seventeenth International Conference on Computers and their Applications, 2002.

[5] Xiaoyan Hong, Mario Gerla and Hanbiao Wang, Loren Clare, "Load Balanced, Energy-Aware Communications for Mars Sensor Networks", "Ad-hoc Network Protocols, IEEEAC, 2002

[6] Namhoon Kim, Jongman Heo, Hyung Seok Kim, Wook Hyun Kwon and, "Reconfiguration of cluster heads for load balancing in wireless sensor networks", Computer Communications, Vol. 31, 2008, pp.153-159.

[7] Vinh Dien Hoang, Zhenhai Shao and Masayuki Fujise, "Efficient Load balancing in MANETs to Improve Network Performance", $6^{\text {th }}$ International Conference on ITS Telecommunications Proceedings, 2006, pp.753756.

[8] D.Sivaganesan and Dr.R.Venkatesan, "Performance Analysis Of Broadcasting In Mobile Ad Hoc Networks Using Cluster Approach", International Journal of Ad hoc, Sensor \& Ubiquitous Computing( IJASUC ) Vol.1, No.2, 2010, pp. 22-31.

[9] Suat Özdemir, "Secure Load Balancing for Wireless Sensor Networks via Inter Cluster Relaying", information security and cryptology conference, turkey,2007, pp. 249-253.

[10] Ioan raicu, Loren Schwiebert, Scott Fowler and Sandeep k.s. Gupta, "Local Load Balancing for Globally Efficient Routing in Wireless Sensor Networks" International Journal of Distributed Sensor Networks, Taylor \& Francis, 2005, pp.163-185.

[11] Zhi Zhang and Guohua Cui, "An Effective Congestion Avoidance Altering Routing Protocol in Sensor Networks", International Conference on Computer Science and Software Engineering, IEEE Computer Soceity, 2008, pp. 980-983.

[12] Santhana Krishnan B and Ramaswamy M, “ A New Cluster Based Protocol For Wireless Sensor Networks", International Conference on Information Science and Application (ICISA-2011), IEEE Computer Society, 2011, pp. 1-8. 